\title{
Effect of the Calibration in Water and the Build-up Cap on the Mg(Ar) Ionization Chamber Measurements
}

\author{
$\underline{\text { H.Koivunoro }}^{1,2,3}$, H. Hyvönen ${ }^{1}$, J. Uusi-Simola ${ }^{4}$, I. Jokelainen ${ }^{5}$, A. Kosunen ${ }^{5}$, M. Kortesniemi' ${ }^{4}$, T. Seppälä3 , I. \\ Auterinen $^{6}$, S. Savolainen ${ }^{2,4}$ \\ ${ }^{1}$ Boneca Corporation, FI-00290 Helsinki, \\ ${ }^{2}$ Department of Physics, POB 64, FI-00014 University of Helsinki, \\ ${ }^{3}$ Department of Oncology, Helsinki University Central Hospital, \\ ${ }^{4}$ HUS Helsinki Medical Imaging Center, University of Helsinki, \\ ${ }^{5}$ Radiation Metrology Laboratory, Radiation and Nuclear Safety Authority-STUK, FI-00881, \\ ${ }^{6}$ VTT Technical Research Centre of Finland, Espoo
}

\begin{abstract}
$\operatorname{Mg}(\mathrm{Ar})$ ionization chamber (IC) is used for photon dose measurements in the epithermal neutron beam of FiR 1 BNCT facility in Finland. Inaccuracy of the chamber measurements remains unsatisfactorily high (up to $10 \%$ ). Previously, the chamber calibrations have been performed in air in the ${ }^{60} \mathrm{Co}$ source at the Radiation Metrology Laboratory of Radiation and Nuclear Safety Authority-STUK, Finland. Build-up caps have been used in the chamber calibration measurements as well as in the phantom measurements in the FiR 1 beam. Aim of this study was to re-evaluate ionization chamber measurement accuracy against simulations at FiR 1 using the new chamber calibration factor defined in water in the ${ }^{60} \mathrm{Co}$ beam. In addition, effect of the build-up cap on the $\operatorname{Mg}(\mathrm{Ar})$ chamber measurements was investigated. The measured absorbed photon dose was compared with the MCNP5 and SERA calculations. Use of build-up cap made no difference on the measured current within statistical uncertainty. When the calibration factor defined in water is applied instead of factor defined in air, the measured dose rate is reduced by $2.6 \%$ and agreement between the measurements and the calculations improves to $0.4 \%-5.0 \%$ at the dose rate maximum, but discrepancy appears along depth in the phantom. At $10 \mathrm{~cm}$ depth, the measurement show about $13 \%-17 \%$ higher photon dose than the calculations. The increasing discrepancy along the depth might suggest that the number of incident photons is underestimated in the FiR 1 beam model and/or that the effective point of the $\mathrm{Mg}(\mathrm{Ar})$ chamber is defined inaccurately. If the sensitive point is moved 0.2 $\mathrm{cm}$ towards tip of the chamber, the difference between measurements and calculations is $5 \%-13 \%$. Remaining difference might be due to inaccurately determined relative sensitivity of the chamber for FiR 1 beam.
\end{abstract}

Keywords: Dosimetry, ionization chamber, SERA, MCNP

\section{Introduction}

$\mathrm{Mg}(\mathrm{Ar})$ ionization chamber (IC) of Exradin is used for photon dose measurements in the epithermal neutron beam of FiR 1 BNCT facility in Finland. The $\mathrm{Mg}(\mathrm{Ar})$ chamber is assumed to be insensitive to neutrons in an epithermal neutron beam (Kosunen et al. 1999). Inaccuracy of the chamber measurements remains unsatisfactorily high (up to $10 \%$ ). Previously, the chamber calibrations have been performed in air in the ${ }^{60} \mathrm{Co}$ source of the Radiation and Nuclear Safety Laboratory of Finland. Build-up cap (BUC) has been applied in the chamber calibration measurements as well as in the phantom measurements on the top of the chamber in order to ascertain the charged particle equilibrium within the chamber wall. Aim of this study was to re-evaluate ionization chamber measurement accuracy against simulations at FiR 1 using the new chamber calibration factor defined in water in the ${ }^{60} \mathrm{Co}$ beam. In addition, effect of the build-up cap on the $\mathrm{Mg}(\mathrm{Ar})$ chamber measurements was investigated.

\section{Materials and Methods}

$\mathrm{Mg}(\mathrm{Ar})$ ionization chamber was calibrated in ${ }^{60} \mathrm{Co}$ source in air and in a water phantom to determine calibration factors $N_{i}$, which defines conversion from detector reading $M$ (current) to dose rate $D_{i}$ in air or water $(=i)$ :

$N_{i}=\frac{\dot{D}_{i}}{M}$

The mass energy absorption coefficient ratios of brain/air (1.107) and brain/water (0.995) defined for the ${ }^{60} \mathrm{Co}$ gamma beam are used to convert the absorbed dose in air or in water further to the 
absorbed dose in brain tissue. In the calibration measurements in air, a $0.2 \mathrm{~cm}$ thick magnesium BUC was used on top of the chamber, while the calibration was performed without the build-up cap in water. At FiR 1, the measurements were performed with and without the build-up cap along the beam central axis in a cylindrical water phantom (diameter $24 \mathrm{~cm})$ and in a cubic $(W \times L \times$ $D: 51 \mathrm{~cm} \times 51 \mathrm{~cm} \times 47 \mathrm{~cm}$ ) water phantom at depths from $1.7 \mathrm{~cm}$ to $14 \mathrm{~cm}$. Chamber stem was aligned along the beam central axis as shown in figure 1 . The geometrical centre of the gas cavity was used as the effective point of the chamber. Measured currents were corrected with ambient temperature and pressure, and normalized according to beam monitor count rate. The measured absorbed photon dose was compared with the MCNP5 (Briesmeister 2000) and SERA (Nigg 1999) calculations. In the calculations, the phantoms were modeled in details. The IC structures were not included in the model and the depth dose distribution was calculated in pure water. The photon dose was calculated using track length estimate of energy deposition tally F6 in units $\mathrm{MeV} / \mathrm{g}$ in MCNP5. The calculation results were normalized according to ratio of measured and calculated ${ }^{197} \mathrm{Au}(\mathrm{n}, \gamma){ }^{198} \mathrm{Au}$ reaction rate at $2 \mathrm{~cm}$ depth in the phantom. For the cylindrical phantom, normalization was performed in the cylindrical PMMA phantom and for the large cubic phantom the same large phantom. The applied normalization factors in the cylindrical phantom were 0.94 for SERA and 0.96 for MCNP5. In the large cubical phantom, the applied normalization factors were 0.99 for SERA and 0.95 for MCNP5.

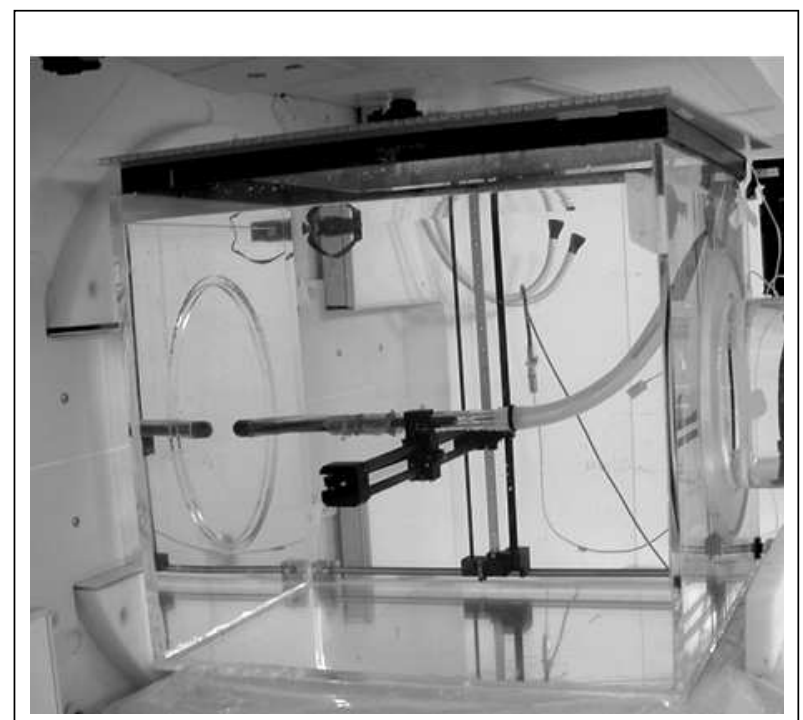

Figure 1. Large cubic ( $W \times \mathbf{X}$ X $D: 51 \mathrm{~cm} \times 51 \mathrm{~cm} \times 47$ $\mathrm{cm})$ water phantom and the $\mathrm{Mg}(\mathrm{Ar})$ ionization chamber at the FiR 1 beam.

\section{Results and Discussions}

The measured photon depth dose distributions in the cylindrical and cubical phantoms are shown in figure 2. The new calibration factor defined in water phantom in ${ }^{60} \mathrm{Co}$ beam was applied in the dose determination. In the phantom measurements with and without the BUC, the measured absolute maximum photon dose rate was found at the $2.9 \mathrm{~cm}$ depth, while no difference was obtained within uncertainty (4\%) in the dose values for the measurement points at the depths from $2 \mathrm{~cm}$ to 3 $\mathrm{cm}$. Similar plateau is obtained for the calculated depth dose rate at the depths from $2.3 \mathrm{~cm}$ to $2.9 \mathrm{~cm}$ in cylindrical phantom with MCNP5 (calculation uncertainty $0.2 \%$ ). Use of build-up cap made no difference on the measured current within measurement uncertainty, obtained difference was $0.2 \%-2.3 \%$. Calibration factor defined in water instead of in air reduces the measured dose rate by $3 \%$ and agreement between the measurements and the calculations improves. This is partly due to fact that the primary quantity has been changed from air kerma or absorbed dose to water. Calculated photon dose deviation from the measured dose is plotted along the phantom depth in figure 3 .
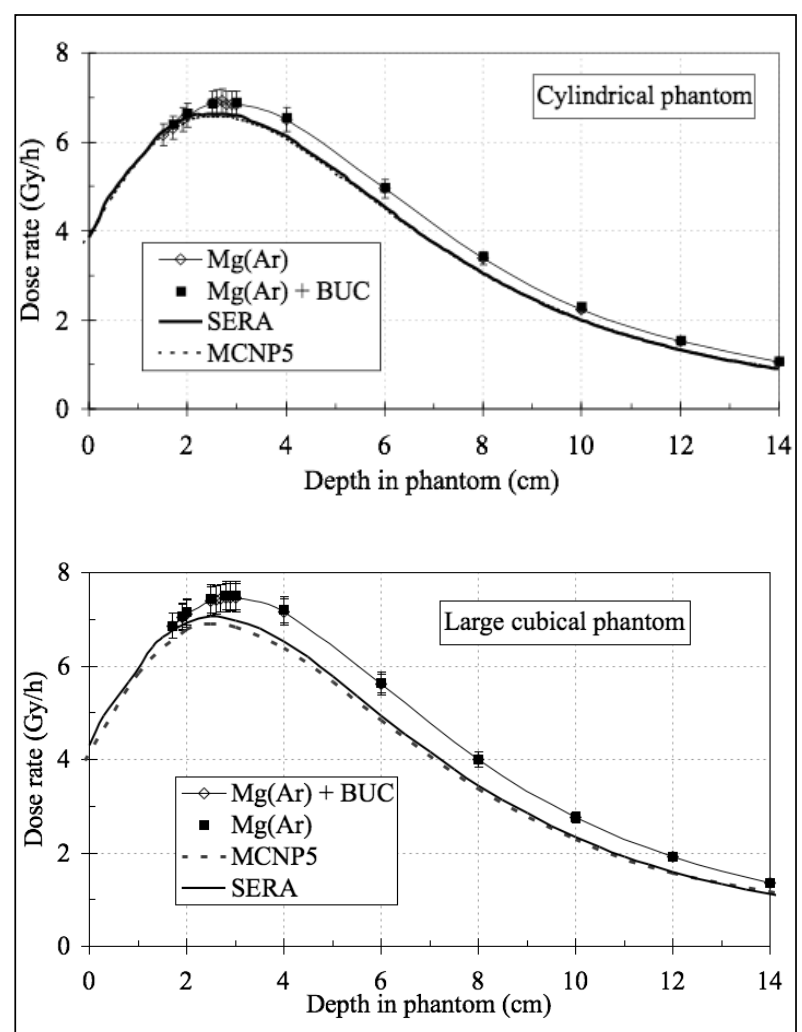

Figure 2. The absorbed photon dose rates measured using the $\mathrm{Mg}(\mathrm{Ar}) \mathrm{IC}$ with the build-up cap $(\mathrm{Mg}(\mathrm{Ar})+\mathrm{BUC})$ and without the cap $(\mathrm{Mg}(\mathrm{Ar}))$ and calculated using SERA and MCNP5 in two water phantoms. The error bar of the measurements is $4 \%$. The statistical error $(1 \sigma)$ of the MCNP5 calculations is $0.2 \%-0.5 \%$ increasing along the phantom depth. 


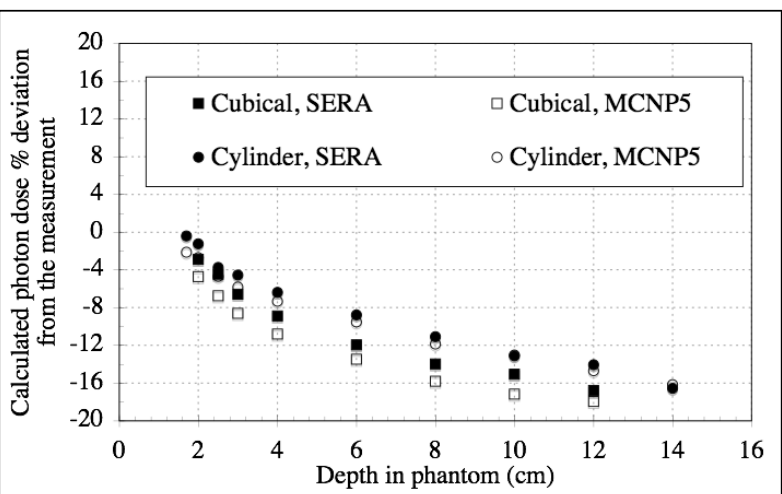

Figure 3. Percentage differences in photon dose rates between calculations and measurements with $\mathrm{Mg}(\mathrm{Ar})$ chamber in the cylindrical and large cubical water phantoms.

Somewhat better agreement between the calculations and the measurements is obtained in the cylindrical phantom. Overall, SERA calculations agree somewhat better with the measurements than MCNP5. The agreement is within $0.4 \%-5.0 \%$ at the dose maximum ( $2 \mathrm{~cm}$ depth), but discrepancy appears along depth in the phantom. At $10 \mathrm{~cm}$ depth, the measurements show about 13.0\%-17.0\% higher photon dose than the calculations. The dose rates determined with SERA and MCNP5 agreed within $2 \%$ at the depths from $0.5 \mathrm{~cm}$ to $14 \mathrm{~cm}$ in the both phantoms. The increasing discrepancy along the depth might suggest that the number of incident photons is underestimated in the FiR 1 beam model and/or that the effective point in the $\operatorname{Mg}(\mathrm{Ar})$ chamber is defined inaccurately. The measurements in the cylindrical phantom require that the chamber stem, and consequently axis of the cylindrical gas cavity, is aligned along the beam central axis (and along the depth dose gradient), not perpendicular like usually in the conventional external photon beam measurements. Since the axis of cylindrical gas cavity is relatively long $(0.9 \mathrm{~cm})$ in comparison to thickness of the cavity $(0.25 \mathrm{~cm})$, the depth dose gradient exists in phantom within the cavity location at almost every measurement point in case of BNCT beam irradiation, and thus location of the sensitive point is more difficult to define. The model presented in ICRU report 26 would give an offset by $1.8 \mathrm{~mm}$ from the geometrical midpoint towards the beam (ICRU 1977). On the other hand the photon field in phantom in case of a neutron beam is mostly created by the induced gammas via the hydrogen capture reactions in water, so the effective point of the chamber may differ from that in an external photon beam measurements and may also depend on the measurement depth. If the sensitive point is moved $0.2 \mathrm{~cm}$ towards tip of the chamber, the difference between measurements and calculations is $5 \%-13 \%$ and with $0.3 \mathrm{~cm}$ move difference is 5\%-11\%. Remaining difference might be due to inaccurately determined relative sensitivity of the chamber for FiR 1 beam. Deviation between the measured and calculated photon dose might also be due to $\mathrm{Mg}(\mathrm{Ar})$ sensitivity to neutrons. Thermal neutron sensitivity of $\mathrm{Mg}(\mathrm{Ar})$ type chamber has reported to increase over time (Raaijmakers et al. 1996). However, at FiR 1, the $\operatorname{Mg}(\mathrm{Ar})$ signal has remained stable in the same measurement geometry for over 10 year (Uusi-Simola 2009).

\section{Conclusions}

Calibration factor defined in water instead of in air in ${ }^{60} \mathrm{Co}$ beam for $\mathrm{Mg}(\mathrm{Ar})$ chamber provides $2.6 \%$ lower photon dose rate in an epithermal neutron beam and improves agreement between the measurements and the calculations. Use of build-up cap with the $\mathrm{Mg}(\mathrm{Ar})$ ionization chamber measurements in water phantom makes no difference on measured signal within measurement uncertainty in the neutron beam. Increasing discrepancy between measured and calculated photon dose rate along the phantom depth may be due to inaccurately determined effective point of the $\mathrm{Mg}(\mathrm{Ar})$ chamber.

\section{References}

Briesmeister, J. F. (Ed.), 2000. MCNP-A General Monte Carlo N Particle Transport Code, Version 4C, LA-13709 M Drake, M.K. 1970 (Data Formats and Procedures for the ENDF Neutron Cross Section Library. BNL-50274 (CT-601)).

ICRU, 1977. Neutron Dosimetry for Biology and Medicine. ICRU Report 26 (ICRU Bethesda, MD).

Kosunen A., Kortesniemi M., Ylä-Mella H., Seppälä T., Lampinen J., Serén T., Auterinen I., Järvinen H. and Savolainen S., 1999. Twin Ionisation Chambers for Dose Determiniations in Phantom in an Epithermal Neutron Beam. Rad. Prot. Dos. 81, 187-194.

Nigg D. W., Wemple C. A., Wcssol D. E. and Wheeler F. J., 1999. SERA - an advanced treatment planning system for neutron therapy and BNCT Trans. ANS 80, 66-8.

Raaijmakers C. P., Watkins P. R., Nottelman E. L., Verhagen H. W., Jansen J. T., Zoetelief J. and Mijnheer B. J., 1996. The neutron sensitivity of dosimeters applied to boron neutron capture therapy. Med. Phys. 23, 1581-9.

Uusi-Simola J, 2009. Finnish dosimetric practice for epithermal neutron beam dosimetry in boron neutron capture therapy. UNIVERSITY OF HELSINKI REPORT SERIES IN PHYSICS (HU-P-D160) https:/oa.doria.fi/bitstream/ handle/10024/44583/finnishd.pdf? sequence $=1$ 